\title{
Uma metodologia para aplicação de estratégias de gamificação na avaliação da aprendizagem de alunos
}

\author{
João Carlos Lima e Silva ${ }^{1}$, \\ Carla Lopes Rodriguez ${ }^{1}$, Rafaela Vilela da Rocha ${ }^{1}$ \\ ${ }^{1}$ Pós-Graduação em Ciência da Computação - Universidade Federal do ABC (UFABC) \\ Santo André - SP - Brazil \\ \{joao.e, c.rodriguez, rafaela.rocha\}@ufabc.edu.br
}

\begin{abstract}
In the educational context the evaluation of learning is a challenge. Several gamified applications have been designed to improve student engagement, but there are still few applications of gamification for assessment. This paper proposes, uses and evaluates the preliminary results of a methodology of application of gamification strategies for student evaluation, through action research. The methodology was used in the gamification of the educational process in two high school classes, total of 71 students, using a developed gammification system. There were benefits of using gamification, such as transparency of results, speed of feedback and student autonomy.
\end{abstract}

Resumo. A avaliação da aprendizagem representa um grande desafio no contexto educacional. Diversas aplicações gamificadas têm sido desenvolvidas para melhorar o engajamento dos alunos, porém ainda são poucas as aplicações da gamificação na avaliação. Este artigo propõe, usa e avalia os resultados preliminares de uma metodologia de aplicação de estratégias de gamificação para avaliação de alunos, por meio de pesquisa-ação. A metodologia foi usada na gamificação do processo educacional em duas turmas de Ensino Médio, total de 71 alunos, por meio do uso de um sistema de avaliação gamificado criado. Verificou-se benefícios do uso da gamificação, como a transparência dos resultados, velocidade de feedback e autonomia dos alunos.

\section{Introdução}

A avaliação é a atividade pela qual podemos medir os resultados do processo de ensinoaprendizagem [Libâneo 2013]. Essa atividade pode ser realizada em diversos momentos do processo, e pode ser classificada, segundo [Bloom et al. 1973], como diagnóstica, formativa e somativa. As formas mais tradicionais de avaliação incluem provas escritas, atividades de pesquisa e trabalhos em grupo. Entretanto, em geral, os alunos não veem as atividades avaliativas como algo positivo [Gehringer and Peddycord 2013].

O interesse pelo tema da gamificação vem crescendo em ritmo acelerado [Kapp 2012]. Essa técnica tem sido usada para melhorar a motivação dos alunos durante e fora das aulas e potencializar o aprendizado. Porém, são poucas as pesquisas e iniciativas que utilizam a gamificação como estratégia de avaliação [Borges et al. 2013]. Sendo que usar elementos de gamificação (por exemplo, pontuação, level e ranking) também pode beneficiar a avaliação dos participantes. 
VIII Congresso Brasileiro de Informática na Educação (CBIE 2019)

Anais do XXX Simpósio Brasileiro de Informática na Educação (SBIE 2019)

Segundo pesquisa da Entertainment Software Association, realizada em 2015, $30 \%$ das pessoas que jogam video game estão na faixa de 18 a 35 anos. O Brasil possui mais de 75 milhões de jogadores e em 2018 era o $13^{\circ}$ no ranking mundial em quantidade de jogadores [Newzoo 2018]. Essa forma de entretenimento tem concorrido com o formato tradicional das aulas. Com o advento da tecnologia móvel e a popularização dos smartphones, tem sido comum alunos concentrarem suas atenções em jogos em momentos que deveriam estar estudando.

O objetivo deste artigo é apresentar a metodologia de aplicação de estratégia de gamificação para avaliação do percurso de aprendizagem, e os resultados preliminares alcançados nas primeiras etapas da pesquisa. Este trabalho é parte de uma pesquisa de mestrado em desenvolvimento, que tem como objetivos investigar como a gamificação pode ser usada para avaliar o aprendizado, desenvolver uma ferramenta de avaliação gamificada, e analisar os seus benefícios, possibilidades e limites quando aplicada em uma turma de Ensino Médio.

O artigo está organizado da seguinte maneira: Na Seção 2 apresentam-se os trabalhos relacionados; na Seção 3 descrevem-se a metodologia utilizada para aplicação de estratégias de gamificação para avaliação de alunos; na Seção 4 apresentam-se os detalhes do sistema web desenvolvido para essa aplicação; os resultados são apresentados na Seção 5 e as considerações finais e trabalhos futuros na Seção 6.

\section{Trabalhos Relacionados}

Existe um grande número de pesquisas sobre aplicação de gamificação na educação [Nah et al. 2014], algumas delas, apesar de não terem seu foco na gamificação como instrumento de avaliação, utilizam em algum momento instrumentos de avaliação em sua metodologia.

[Tenório et al. 2016] realizaram um estudo sobre o uso de gamificação em um sistema de avaliação por pares. Foi identificado que melhorar a motivação dos alunos ao avaliar os trabalhos dos seus pares tem como resultado a diminuição do tempo de correção. Segundo os autores, a gamificação trouxe uma melhoria na participação dos alunos-avaliadores nos estudos realizados.

[Kocadere and Şeyma Çağlar 2015] aplicaram a gamificação em um sistema de avaliação. O sistema utiliza diversos elementos de gamificação como avatar, níveis e badges. Após a aplicação foram levantados os dados através de entrevistas e questionários e concluíram que o uso da gamificação na avaliação trouxe mais motivação e prazer na realização dos exames, além de reduzir a ansiedade, característica dos testes.

[Gehringer and Peddycord 2013] realizaram uma pesquisa trocando o sistema de notas percentuais pelo sistema de pontos, no qual o aluno deixa de perder nota por não realizar uma atividade e passa a ganhar pontos por cada atividade realizada, podendo inclusive ganhar pontos extras ao realizar avaliação por pares. Como resultado, os autores perceberam que os alunos se sentiram mais motivados em realizar as atividades e as avaliações por pares no sistema de pontuação.

[Epema and Iosup 2014] aplicaram a gamificação em um curso completo de Arquitetura de Computadores. Os resultados foram um aumento no número de alunos aprovados na disciplina, e um maior envolvimento dos alunos para realização de atividades e 
VIII Congresso Brasileiro de Informática na Educação (CBIE 2019)

Anais do XXX Simpósio Brasileiro de Informática na Educação (SBIE 2019)

participação em sala de aula.

O presente trabalho avança os estudos sobre o engajamento iniciados por [Tenório et al. 2016] e [Epema and Iosup 2014], aplicando diferentes estratégias em outro contexto (Ensino Médio). Além disso, apropria-se das técnicas propostas por [Kocadere and Şeyma Çağlar 2015] e [Gehringer and Peddycord 2013] para integrar os benefícios da gamificação na avaliação do aprendizado, focando na análise dos resultados dessas avaliações.

\section{Metodologia}

Conforme apresentado na Introdução, o objetivo do presente trabalho é apresentar a metodologia adotada para aplicação das estratégias de gamificação na avaliação da aprendizagem de alunos, e os resultados preliminares alcançados nas primeiras etapas da pesquisa realizada.

Visando atingir o objetivo proposto, realizou-se uma pesquisa-ação na disciplina de Informática Aplicada, no Ensino Médio Integrado. Por haver envolvimento do pesquisador no processo de investigação, uma vez que o pesquisador é também professor da disciplina Informática Aplicada, onde ocorreu a realização do experimento, considera-se a metodologia pesquisa-ação como a opção mais adequada para a presente investigação, ou pesquisa participante, conforme definição de [Demo 1995]. Esse tipo de pesquisa se caracteriza por ser realizada em ciclos de Planejamento, Ação e Reflexão. Na fase de planejamento é identificado o problema e desenhada uma possível solução, na fase de ação a solução proposta é colocada em prática e o ambiente é observado, finalmente, na fase de reflexão os resultados são avaliados e comparados com a teoria. Após a fase de reflexão os resultados são divulgados e o ciclo pode se repetir até que o objetivo seja alcançado [Filippo et al. 2018].

A investigação foi realizada com duas turmas presenciais da primeira série do Ensino Médio na disciplina de Informática Aplicada, sendo uma turma do Ensino Médio Integrado ao Técnico em Química, e a outra do Ensino Médio Integrado ao Técnico em Administração. Foram selecionadas duas turmas, para aumentar a amostra populacional, sendo assim, a pesquisa investiga os efeitos na população de 80 alunos, 40 de cada curso. Apesar da diferença entre as bases profissionalizantes, as duas turmas estão na mesma faixa etária e a disciplina de Informática Aplicada ministrada nas duas turmas possui os mesmos objetivos, competências e habilidades, independente do curso.

[Kapp 2013] divide a gamificação em dois tipos: gamificação estrutural e gamificação do conteúdo. Na gamificação estrutural o conteúdo apresentado permanece inalterado e a estrutura de interação, feedback e recompensa utiliza os elementos de gamificação. Na gamificação de conteúdo, por sua vez, o conteúdo do curso é modificado para tornar-se gamificado, nesse caso pode-se utilizar recursos de storytelling para tornar o conteúdo mais dinâmico. A presente pesquisa iniciou a investigação através da gamificação estrutural e avançou até a gamificação do conteúdo. Portanto, foi dividida em três momentos, com cenários distintos para as duas turmas, conforme Quadro 1.

O Cenário 1 (primeiro bimestre) foi aplicado no primeiro bimestre do curso, onde a aula seguiu uma abordagem tradicional, com aulas expositivas e avaliações através de questionários fechados e exercícios práticos em laboratório de informática. No Cenário 2 
VIII Congresso Brasileiro de Informática na Educação (CBIE 2019)

Anais do XXX Simpósio Brasileiro de Informática na Educação (SBIE 2019)

\begin{tabular}{l|l|l|l} 
Cenário & Gamificação & Avaliação & Período \\
\hline Cenário 1 & Não Aplicada & Tradicional & $1^{\circ}$ Bimestre (fevereiro-abril/2019) \\
Cenário 2 & Estrutural & Gamificada & $2^{\circ}$ Bimestre (maio-julho/2019) \\
Cenário 3 & Estrutural e de Conteúdo & Gamificada & $3^{\circ}$ Bimestre (agosto-outubro/2019)
\end{tabular}

Quadro 1: Cenários de aplicação da gamificação

(segundo bimestre), foi aplicada a gamificação estrutural e os alunos foram avaliados utilizando elementos de gamificação, porém as aulas seguiram a mesma didática do Cenário 1. Um sistema web gamificado foi utilizado para dar suporte às avaliações, que ocorreram durante todo bimestre. O Cenário 3 (previsto para ser aplicado no terceiro bimestre) irá introduzir a gamificação do conteúdo, além da estrutural, substituindo a aula expositiva por missões que devem ser cumpridas através de pesquisa e interações entre os alunos com suporte do professor. Da mesma forma que no Cenário 2, a avaliação será gamificada com suporte do sistema web desenvolvido.

Nos três cenários são colhidos dados para análises de desempenho das estratégias e comparação entre os cenários utilizados, conforme Quadro 2.

\begin{tabular}{l|l} 
Variável & Instrumento \\
\hline Engajamento & Questionário de Escala de Engajamento \\
Perfil de Aprendizagem & Questionário VARK \\
Perfil de Jogador & Questionário de Marczewski
\end{tabular}

Quadro 2: Variáveis e instrumentos de coleta de dados

Para analisar o engajamento dos alunos nos dois primeiros cenários, foi aplicado um questionário de medida de engajamento, proposto inicialmente por [Schaufeli and Bakker 2003] com 17 questões, onde o participante responde a cada questão indicando valores em uma escala de 0 a 6 . O referido questionário foi, posteriormente, encurtado pelo próprio autor para 9 questões [Bakker et al. 2006] usadas nesta pesquisa. Devido ao fato do questionário proposto ter o objetivo de avaliar o engajamento do aluno frente à escola, a redação das questões foi adaptada para o contexto do componente curricular. Esse questionário foi aplicado ao final de cada um dos cenários.

De forma complementar, foi realizada uma avaliação sobre o perfil de aprendizagem de cada aluno no início do ano letivo. Esses dados foram utilizados para uma análise comparativa, visando compreender a eficácia de cada estratégia de gamificação em relação a cada perfil de aprendizagem. Para tanto, foi aplicado o questionário VARK, que possui 16 questões e aponta o perfil de aprendizagem de acordo com quatro características: visual, auditivo, leitor/escritor e cinestésico [Fleming 2001]. Outra análise realizada foi a identificação do perfil de jogador de cada aluno, com a finalidade de analisar a efetividade da aplicação gamificada para cada estilo de jogador. Para isso, foi aplicado um questionário composto de 24 questões com respostas no formato de escala Likert de cinco pontos para classificação dos alunos de acordo com a tipologia de [Marczewski 2015], que enquadra cada jogador em seis possíveis perfis: achiever, disruptivo, espírito livre, filantropo, jogador e social.

Com os dados obtidos durante a etapa de levantamento de dados foram realizadas 
VIII Congresso Brasileiro de Informática na Educação (CBIE 2019)

Anais do XXX Simpósio Brasileiro de Informática na Educação (SBIE 2019)

comparações para identificar correlação entre as estratégias de gamificação e os perfis (de aprendizagem e de jogador) dos alunos.

\section{Sistema de Avaliação gamificado}

O sistema desenvolvido é uma plataforma web gamificada para apoio à avaliação dos alunos. Ele foi utilizado a partir do Cenário 2. A Figura 1 mostra a tela inicial do sistema onde os alunos podem ver o resultado atual do seu desempenho na disciplina e realizar as avaliações.

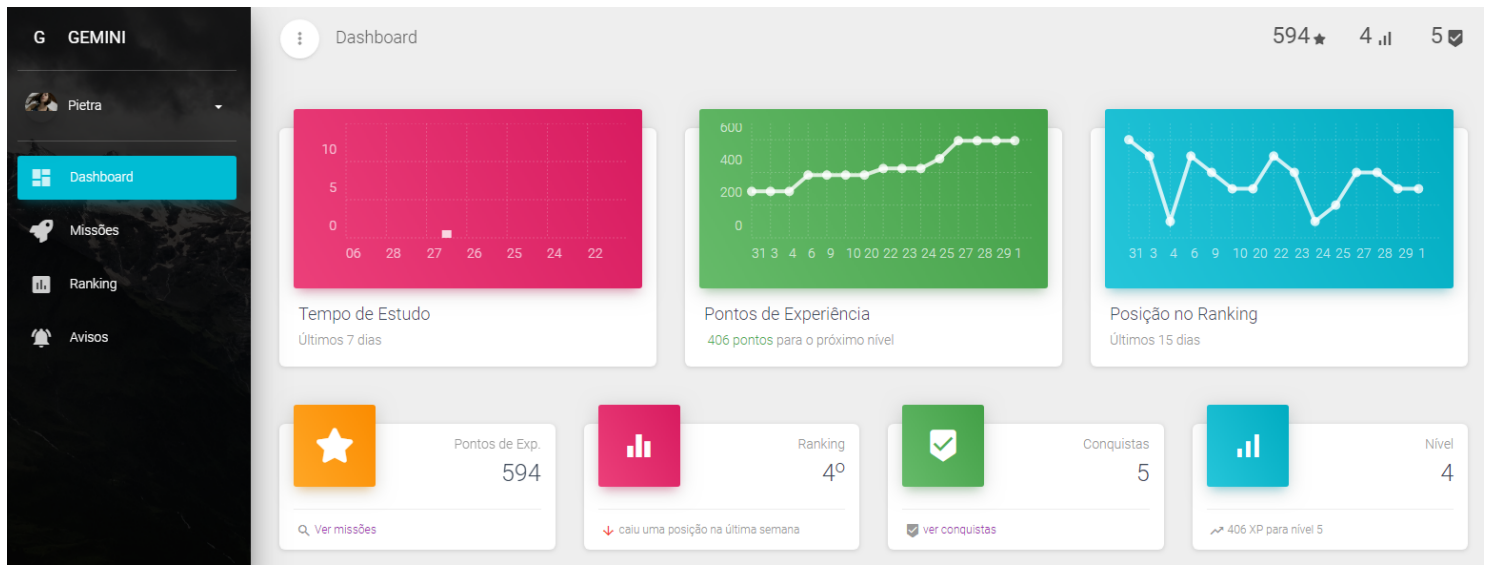

Figura 1. Dashboard do Sistema de Avaliação Gamificado

No sistema os alunos podem visualizar o seu desempenho através de quatro variáveis:

- Pontos de Experiência: ao cumprir uma missão no sistema o aluno é pontuado com pontos de experiência de acordo com o desempenho dele na atividade. Para passar de nível é necessário alcançar uma quantidade pré-determinada de pontos de experiência.

- Nível: para ter acesso às missões avançadas os alunos precisam alcançar um determinado nível. Esse recurso possibilita um crescimento contínuo da dificuldade da avaliação, ou seja, um aluno só pode realizar uma atividade mais complexa depois ter realizado de modo satisfatório as atividades mais simples.

- Ranking: representa a posição do aluno no ranking da sala para comparativo do desempenho dos colegas. Cada aluno pode ver apenas os cinco primeiros colocados e a sua posição no ranking, dessa forma os melhores classificados são estimulados sem expor os últimos colocados.

- Conquistas: avaliação qualitativa das competências dos alunos. O professor atribui uma conquista para o aluno conforme ele demonstra, em sala de aula ou em alguma atividade, a aquisição de uma competência prevista no plano de curso.

O sistema permite que os alunos cumpram missões realizando as fases definidas pelo professor. Uma missão é composta por diversas fases, essas fases podem ser uma atividade prática ou um questionário. A pontuação das fases e um feedback de texto dado pelo professor ficam disponíveis para os alunos.

No mesmo sistema os alunos respondem três questionários: perfil de aprendizagem, perfil de jogador e engajamento (Figura 2). Ao responder esses questionários eles também são premiados com pontos de experiências. 
VIII Congresso Brasileiro de Informática na Educação (CBIE 2019)

Anais do XXX Simpósio Brasileiro de Informática na Educação (SBIE 2019)

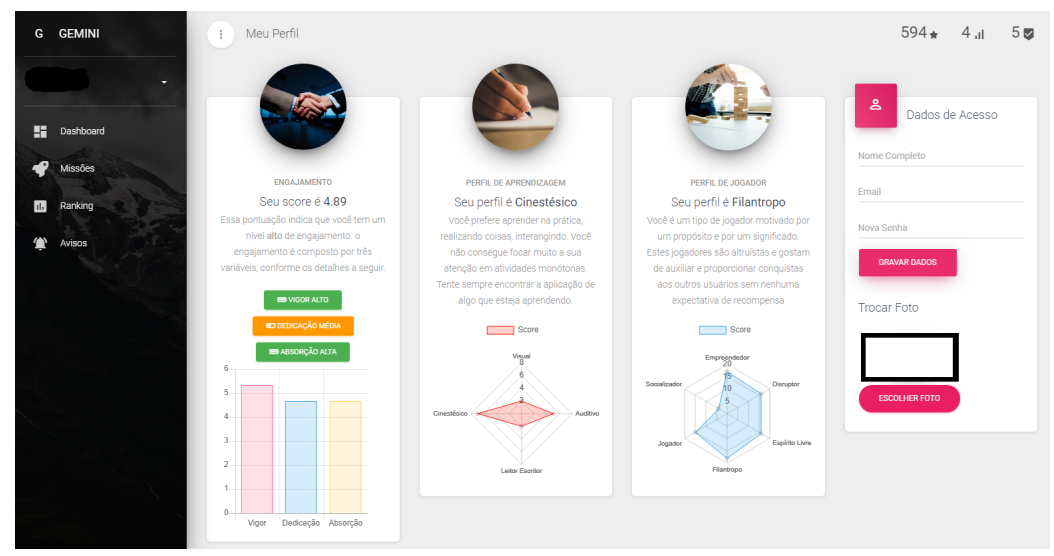

Figura 2. Perfil dos Alunos com questionários respondidos

\section{Resultados Preliminares}

Para análise dos resultados foram realizadas comparações entre os dados colhidos inicialmente nos cenários 1 e 2 .

A primeira análise realizada refere-se ao engajamento dos alunos nos dois cenários. O engajamento foi calculado através das respostas do questionário de engajamento proposto por [Bakker et al. 2006]. Os questionários foram aplicados ao final de cada cenário. Conforme representado no gráfico da Figura 3 houve um pequeno aumento no engajamento dos alunos com a utilização do sistema gamificado. Podemos inferir que essa pequena variação média $(3,7 \%)$ se deve ao fato de alguns alunos apresentarem um engajamento menor no segundo cenário, enquanto outros ficaram mais engajados no novo ambiente.

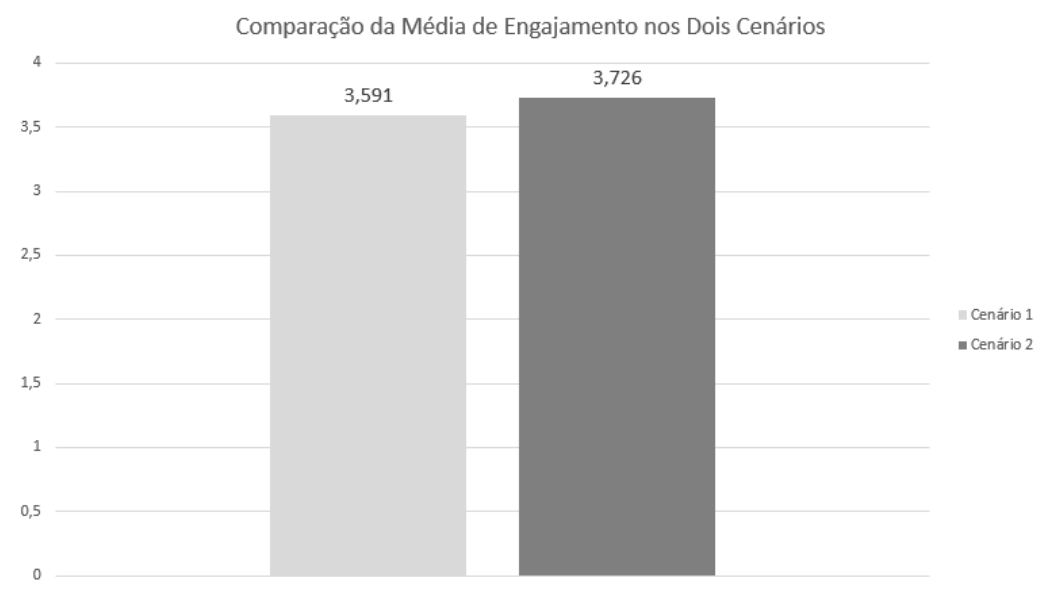

Figura 3. Comparação do engajamento

Para melhor compreensão desse resultado inicial, foi realizada uma análise específica de acordo com os estilos de jogador de cada aluno. O gráfico da Figura 4 indica que a gamificação aplicada no cenário 2 refletiu em um maior engajamento dos alunos com estilo de jogador socializador (aumento de 12,4\%) e empreendedor (aumento de $5,2 \%)$. 
VIII Congresso Brasileiro de Informática na Educação (CBIE 2019)

Anais do XXX Simpósio Brasileiro de Informática na Educação (SBIE 2019)

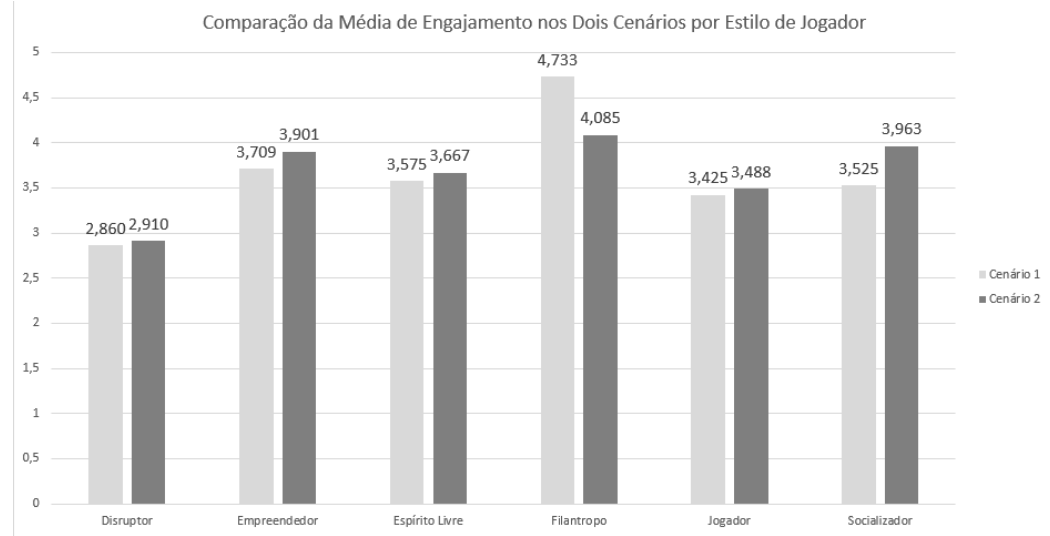

Figura 4. Comparação do engajamento por estilo de jogador

O jogador com estilo empreendedor busca sempre aprender coisas novas e é motivado por desafios. O sistema proposto incluiu o desafio na dinâmica da sala de aula, motivando o aluno a superar as dificuldades para aprender. O jogador com estilo socializador é motivado pelas relação com os colegas, observa-se que essa evolução no engajamento desses alunos tenha se dado pelo elemento ranking do sistema, que motivou esses alunos a buscarem as primeiras posições para se destacar diante dos colegas. Por sua vez, alunos com o estilo de jogador filantropo, que por definição tem a característica de ajudar os demais jogadores e não ser motivado por recompensas pessoais, tiveram uma redução de $13,7 \%$ no engajamento, pois o sistema proposto não possibilita que os alunos colaborem entre si.

Para entender como os diferentes estilos de aprendizagem são afetados pelo sistema de avaliação gamificado, foi realizada uma comparação com os resultados do engajamento dos alunos de acordo com o perfil avaliado pelo questionário VARK. O gráfico da Figura 5 mostra que alunos com perfil cinestésico tiveram um aumento de 8,6\% no engajamento, alunos com perfil visual tiveram um aumento de $9,3 \%$, enquanto aqueles que possuem o perfil auditivo apresentaram um engajamento $8,9 \%$ abaixo em relação ao primeiro cenário.

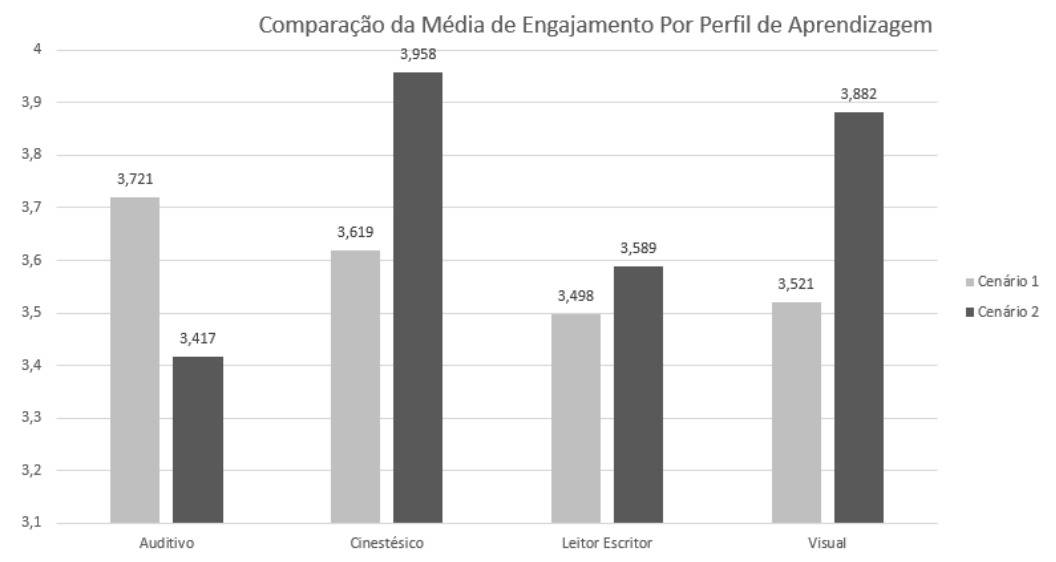

Figura 5. Comparação do engajamento por perfil de aprendizagem

Segundo [Fleming 2001], alunos com perfil cinestésico aprendem melhor pela 
VIII Congresso Brasileiro de Informática na Educação (CBIE 2019)

Anais do XXX Simpósio Brasileiro de Informática na Educação (SBIE 2019)

prática. O sistema proposto tornou a experiência da aula mais prática, pois os alunos tinham que realizar diversas atividades para cumprir as missões. Por sua vez, pessoas com perfil visual aprendem melhor vendo e desenhando. Esses alunos foram beneficiados com a redução de aulas expositivas e com a inserção do sistema que possibilitou a visualização de vídeos, gráficos e demonstrações das atividades que deveriam ser realizadas. Em contrapartida, alunos com o perfil auditivo, que aprendem melhor ao ouvir, dialogar e debater, tiveram seu engajamento reduzido pelo fato do novo cenário privilegiar a prática em detrimento da exposição teórica do conteúdo.

Vale ressaltar que nem todos os alunos responderam todos os questionários. Os alunos que não tinham todos os dados completos não foram considerados nessa análise. O total de alunos com dados completos foi de 71.

Finalmente uma última análise foi realizada de forma empírica, baseada na experiência do professor em sala de aula. Após a introdução do sistema gamificado na aula de Informática Aplicada, observou-se três benefícios claros em relação à aula tradicional. Primeiramente, os alunos deixaram de ter uma postura passiva, esperando que o professor apresentasse o conteúdo e indicasse a atividade que deveria ser feita. Eles passaram a ter a iniciativa de resolver os problemas propostos no sistema, e quando terminavam cobravam do professor novas atividades para que pudessem ganhar mais pontos.

Um segundo benefício em relação à avaliação da aprendizagem foi a transparência e velocidade da divulgação dos resultados. Todos os dias os alunos tomavam contato com o resultado do seu desempenho na disciplina, verificando sua pontuação e nível no sistema. Dessa forma, poderiam tomar ações para superar dificuldades em temas específicos antes do término do bimestre, quando fatalmente seria tarde demais.

De forma não proposital, o sistema permitiu a evolução progressiva das atividades avaliativas. Alunos que não conseguiram atingir a pontuação mínima necessária para acessar as avaliações do nível 2 eram obrigados a refazer atividades do nível 1. Esse mecanismo permitiu uma recuperação contínua e personalizada dos alunos com maior dificuldade.

\section{Conclusões e Trabalhos Futuros}

Esse artigo apresentou uma metodologia para aplicação de estratégias de gamificação para avaliação de alunos duas turmas da disciplina Informática Aplicada do Ensino Médio. Entre as contribuições do trabalho podemos destacar a análise de melhoria do engajamento por estilo de jogador e perfil de aprendizagem. Essa análise mostrou-se útil para guiar as ações do professor no sentido de obter os benefícios da gamificação para alunos que não haviam sido atingidos previamente. Além disso, foi possível demonstrar a viabilidade da estratégia para a avaliação da aprendizagem e listar as experiências positivas nos cenários 1, mais tradicional, e 2, com elementos gamificados.

Até o momento, a metodologia de aplicação da ferramenta de avaliação gamificada mostrou diversos benefícios para a disciplina. Entre os quais podemos citar a melhoria do engajamento dos alunos, a velocidade do feedback de desempenho das avaliações e a autonomia dos alunos para realização das atividades.

O sistema gamificado desenvolvido permitiu que os alunos visualizassem os seus resultados e, de forma comparativa através do ranking, tomassem ciência do seu desempe- 
VIII Congresso Brasileiro de Informática na Educação (CBIE 2019)

Anais do XXX Simpósio Brasileiro de Informática na Educação (SBIE 2019)

nho na disciplina. Além disso, diferente da avaliação tradicional, quando aplicada apenas ao fim de um processo, o professor pôde identificar antecipadamente alunos que necessitavam de ações especiais para atingir os objetivos de aprendizagem.

Algumas limitações do sistema foram encontradas, como a redução do engajamento em alguns alunos de perfil de aprendizagem e de jogador específicos. Em relação aos alunos com estilo de jogador filantropo, por exemplo, pode-se atribuir uma pontuação para alunos que ajudem colegas com mais dificuldade. Dessa forma, entende-se que esses alunos se sentiriam mais engajados com a gamificação. Para alunos com perfil de aprendizagem auditivo, é possível incluir no sistema atividades de debate e discussões.

Esse trabalho é parte de uma pesquisa de mestrado em andamento. Os cenários 1 e 2 foram aplicados e analisados considerando, principalmente, o engajamento e o desempenho dos alunos nas atividades propostas. O cenário 3 ainda será realizado para ter seus dados analisados de forma a complementar os resultados e discussões apresentados até aqui. Essa complementação envolve, por exemplo, analisar e comparar o desempenho acadêmico dos alunos nos três cenários, incluindo a avaliação qualitativa realizada pelo professor.

Essa pesquisa limitou-se a aplicação em duas turmas de Ensino Médio. Os alunos, apesar de perfis diferentes, estão na mesma faixa etária e escolaridade. Uma possibilidade de trabalhos futuros é a aplicação em turmas de outros níveis para compreensão dos resultados da avaliação gamificada em alunos do ensino fundamental, profissionalizante e superior.

\section{Referências}

Bakker, A. B., Schaufeli, W. B., Bakker, A. B., and Salanova, M. (2006). The Measurement of Short Questionnaire A Cross-National Study. In Educational and Psychological Measurement, volume 66, pages 701-716.

Bloom, B. S., Engelhart, M. D., Furst, E. J., Hill, W. H., and Krathwohl (1973). Taxionomia dos objetivos educacionais. Globo, Porto Alegre.

Borges, S. S., Reis, H. M., Durelli, V. H. S., Bittencourt, I. I., Jaques, P. A., and Isotani, S. (2013). Gamificação aplicada à educação: um mapeamento sistemático. In Simpósio Brasileiro de Informática na Educação - SBIE.

Demo, P. (1995). Metodologia científica em ciências sociais. Atlas, São Paulo, $3^{\text {a }}$ edition.

Epema, D. and Iosup, A. (2014). An experience report on using gamification in technical higher education. In ACM Technical Symposium on Computer Science Education SIGCSE.

Filippo, D., Roque, G., and Pedrosa, S. (2018). Pesquisa-ação: possibilidades para a informática educativa. Livro CBIE.

Fleming, N. D. (2001). Teaching and Learning Styles: VARK Strategies. IGI Global.

Gehringer, E. and Peddycord, B. (2013). Grading by experience points: An example from computer ethics. Proceedings - Frontiers in Education Conference, FIE, pages $1545-1550$.

Kapp, K. M. (2012). The Gamification of learning and instruction: Game-based methods and strategies for training and education. Pfeiffer. 
VIII Congresso Brasileiro de Informática na Educação (CBIE 2019)

Anais do XXX Simpósio Brasileiro de Informática na Educação (SBIE 2019)

Kapp, K. M. (2013). The gamification of learning and instruction - fieldbook. Ideas into practice. Pfeiffer.

Kocadere, S. A. and Şeyma Çağlar (2015). The Design and Implementation of a gamified Assessment. In Journal of e-Learning and Knowledge Society, volume 11. JLKS.

Libâneo, J. C. (2013). Didática. Cortez.

Marczewski, A. (2015). Even Ninja Monkeys Like to Play: Gamification, Game Thinking \& Motivational Design. Blurb.

Nah, F. F.-H., Zeng, Q., Telaprolu, V. R., Ayyappa, A. P., and Eschenbrenner, B. (2014). Gamification of Education: A Review of Literature. HCI in Business.

Newzoo (2018). Global Games Market Report. Technical report, Newzoo.

Schaufeli, W. B. and Bakker, A. B. (2003). Test manual for the Utrecht Work Engagement Scale. Unpublished manuscript, Utrecht University, the Netherlands.

Tenório, T., Bittencourt, I. I., Isotani, S., Pedro, A., and Ospina, P. (2016). A gamified peer assessment model for on-line learning environments in a competitive context. In Computer in Human Behavior, volume 64. 